\title{
BMS group at spatial infinity: the Hamiltonian (ADM) approach
}

\author{
Marc Henneaux ${ }^{a, b}$ and Cédric Troessaert ${ }^{c}$ \\ ${ }^{a}$ Université Libre de Bruxelles and International Solvay Institutes, \\ ULB-Campus Plaine CP231, B-1050 Brussels, Belgium \\ ${ }^{b}$ Collège de France, \\ 11 place Marcelin Berthelot, 75005 Paris, France \\ ${ }^{c}$ Max-Planck-Institut für Gravitationsphysik (Albert-Einstein-Institut), \\ Am Mühlenberg 1, DE-14476 Potsdam, Germany \\ E-mail: henneaux@ulb.ac.be, ctroessaert@aei.mpg.de
}

ABSTRACT: New boundary conditions for asymptotically flat spacetimes are given at spatial infinity. These boundary conditions are invariant under the BMS group, which acts non trivially. The boundary conditions fulfill all standard consistency requirements: (i) they make the symplectic form finite; (ii) they contain the Schwarzchild solution, the Kerr solution and their Poincaré transforms, (iii) they make the Hamiltonian generators of the asymptotic symmetries integrable and well-defined (finite). The boundary conditions differ from the ones given earlier in the literature in the choice of the parity conditions. It is this different choice of parity conditions that makes the action of the BMS group non trivial. Our approach is purely Hamiltonian and off-shell throughout.

Keywords: Classical Theories of Gravity, Global Symmetries, Space-Time Symmetries

ARXIV EPRINT: 1801.03718 


\section{Contents}

1 Introduction 1

2 Background $\quad 3$

2.1 Fall-off at spatial infinity - RT parity conditions 3

2.2 Spherical coordinates 5

3 New boundary conditions $\quad 8$

3.1 Explicit form 8

3.2 Constraints 9

3.3 Preservation under surface deformations 9

4 Asymptotic charges $\quad 10$

$\begin{array}{lll}5 & \text { The BMS algebra } & 13\end{array}$

6 Conclusions $\quad 15$

A Radial decomposition of the spatial metric and the spatial curvature $\quad 16$

B More details on the divergences of Lorentz generators 18

C BMS algebra in the "instant form" and in the hyperbolic form of the $\begin{array}{ll}\text { dynamics } & 20\end{array}$

\section{Introduction}

In relativistic quantum field theory, the states of the quantum fields are defined on Cauchy surfaces, which one usually takes to be spacelike hyperplanes. A Lorentz observer is characterized by the family of hyperplanes containing events simultaneous to the observer at the "same" time, which are parallel to the simultaneity hyperplane at a fixed given time, $x^{0}=0$, say. The dynamical evolution determines how the physical state changes as one moves from one spacelike hyperplane to the next. This is the "instant form" of the dynamics in the language of [1]. The Poincaré transformations preserving the foliation slice by slice are the kinematical transformations (spatial translations and spatial rotations), while the other Poincaré generators are dynamical (and called the "Hamiltonians" in [1]).

In the presence of gravitation, foliations by spacelike hyperplanes are not available. In the asymptotically flat case, however, this structure appears at infinity [2], a fact made particularly clear in the Hamiltonian formulation of general relativity [3-5]. The Poincaré group structure in Dirac's "instant form" was exhibited in the pioneering paper [6], where 
precise boundary conditions at spatial infinity were given and shown to yield the Poincaré algebra as asymptotic symmetry algebra.

The asymptotic structure at null infinity was studied in [7-12] and shown to be invariant under an infinite dimensional algebra now called the "BMS" algebra (for a recent review, see $[13,14])$. The enlargement of this algebra by "super-rotations" was more recently performed in [15-24] where the Lorentz algebra is extended to the conformal algebra in 2 dimensions while even bigger enlargements where also proposed in [25-28]. The remarkable potential physical implications of the BMS algebra both for the infrared structure of gravity [29-38] and for black hole physics [39-45] have attracted considerable interest in the last years [46].

It is implicit in this exciting work that the BMS algebra is realized in the quantum theory in terms of charges acting in the Hilbert space of states of the theory. These charges should have an expression at spatial infinity in the ADM formulation of the evolution based on foliations that become asymptotically parallel hyperplanes, corresponding to inertial observers at infinity. However, the boundary conditions adopted in [6] at spatial infinity to make the angular momentum finite also make all BMS charges identically vanishing. Technically, as shown in [6], this is a consequence of the so-called parity conditions imposed on the leading order of the metric and its conjugate momentum as one recedes to spatial infinity. In order to resolve this tension between the asymptotic structure at spatial infinity and the BMS algebra emerging at null infinity, one must adopt boundary conditions at spatial infinity different from those of [6].

One cannot just drop the standard parity conditions, since the symplectic structure, the angular momentum and the "boost charges" generically diverge logarithmically without them [47]. One must therefore find alternative conditions that preserve finiteness and, at the same time, leave room for a well-defined and non trivial action of the BMS algebra.

We propose in this paper new boundary conditions at spatial infinity that fulfill this purpose. These boundary conditions (i) are invariant under the BMS algebra, (ii) make the symplectic form finite, (iii) contain the Schwarzchild solution, the Kerr solution and their Poincaré transforms, and (iv) make the Hamiltonian generators of the asymptotic symmetries integrable, well-defined (finite) and generically non-zero.

The new consistent boundary conditions given here involve parity conditions of a different type than those of [6]. The existence of alternative parity conditions making the symplectic structure finite was observed in the insightful work [48], but their full consistency was not studied. The work [48] went indeed in a somewhat orthogonal direction since it was concerned with relaxing the parity conditions altogether and dealing with the ensuing divergences through holographic renormalisation. Nevertherless, the analysis of [48] and the subsequent developments of [49] on the structure of its asymptotic symmetry, were important for arriving at the new boundary conditions proposed in this paper.

Our work is organized as follows. In section 2, we recall some classic background information on the ADM Hamiltonian treatment of asymptotically flat spacetimes with the parity conditions of [6]. This is necessary to motivate and derive our results. We formulate the asymptotic conditions both in asymptotically cartesian and asymptotically spherical coordinates, as it turns out that the new boundary conditions are most conve- 
niently expressed in asymptotically spherical coordinates. Next, in section 3, we give the explicit form of the new boundary conditions and verify that they consistently contain the Schwarzschild solution, the Kerr solution, and their Poincaré transforms. We also work out the form of the asymptotic symmetries. In section 4, we prove that the Hamiltonian generators of the asymptotic symmetries are integrable and finite. We also point out that the charges associated with supertranslations need not vanish. Section 5 is devoted to showing that the Hamiltonian generators of the asymptotic symmetries close according to the BMS algebra. Finally, section 6 summarizes our results and comments on various possible directions for extending them. Three technical appendices complete our paper.

We focus here on vacuum gravity. Furthermore, our analysis is carried out in the Hamiltonian formalism of [3-5] throughout. The boundary conditions are expressed on the canonical variables " $(q, p)$ " at any given time. The action to be used in the path integral is $\int(p \dot{q}-H) d t$ where $q(t)$ and $p(t)$ fulfill at all $t$ 's the boundary conditions given in this paper but are not assumed to obey the equations of motion. There are also Lagrange multipliers in the action, which must define asymptotic symmetries, i.e., define transformations that preserve the boundary conditions.

\section{Background}

\subsection{Fall-off at spatial infinity - RT parity conditions}

Our starting point are the standard Hamiltonian boundary conditions for asymptotically flat spacetimes, given on spatial slices that asymptote hyperplanes equipped with asymptotically cartesian coordinates $x^{i}=(x, y, z)$ at spatial infinity $\left(r \rightarrow \infty\right.$ with $\left.r^{2}=x^{i} x_{i}\right)$. On any such hypersurface, the spatial metric $g_{i j}$ and its conjugate momentum $\pi^{i j}$ behave as

$$
\begin{aligned}
g_{i j} & =\delta_{i j}+\frac{1}{r} \bar{h}_{i j}+\frac{1}{r^{2}} h_{i j}^{(2)}+o\left(r^{-2}\right), \\
\pi^{i j} & =\frac{1}{r^{2}} \bar{\pi}^{i j}+\frac{1}{r^{3}} \pi^{(2) i j}+o\left(r^{-3}\right) .
\end{aligned}
$$

Indices are lowered and raised with the background flat metric $\delta_{i j}$ and its inverse. The coefficients in both expansions are functions on the unit sphere. We adopt the general convention that barred quantities, such as $\bar{h}_{i j}$ or $\bar{\pi}^{i j}$ are functions on the unit sphere and so are $O(1)$. The boundary conditions include the Schwarzschild and Kerr metrics.

Under a deformation of the constant time hypersurface parametrized by $\left(\xi^{\perp} \equiv \xi, \xi^{i}\right)$, the canonical variables transform as $[3,5]$

$$
\begin{aligned}
\delta g_{i j}= & 2 \xi g^{-\frac{1}{2}}\left(\pi_{i j}-\frac{1}{2} g_{i j} \pi\right)+\mathcal{L}_{\xi} g_{i j}, \\
\delta \pi^{i j}= & -\xi g^{\frac{1}{2}}\left(R^{i j}-\frac{1}{2} g^{i j} R\right)+\frac{1}{2} \xi g^{-\frac{1}{2}}\left(\pi_{m n} \pi^{m n}-\frac{1}{2} \pi^{2}\right) \\
& -2 \xi g^{-\frac{1}{2}}\left(\pi^{i m} \pi_{m}{ }^{j}-\frac{1}{2} \pi^{i j} \pi\right)+g^{\frac{1}{2}}\left(\xi^{i j}-g^{i j} \xi^{\mid m}{ }_{\mid m}\right) \\
& +\mathcal{L}_{\xi} \pi^{i j}
\end{aligned}
$$


where $\mathcal{L}_{\xi} g_{i j}$ and $\mathcal{L}_{\xi} \pi^{i j}$ are respectively the Lie derivatives of $g_{i j}$ and $\pi^{i j}$ along the vector field $\xi^{i}$,

$$
\begin{aligned}
\mathcal{L}_{\xi} g_{i j} & =\xi_{i \mid j}+\xi_{j \mid i}, \\
\mathcal{L}_{\xi} \pi^{i j} & =\left(\pi^{i j} \xi^{m}\right)_{\mid m}-\xi^{i}{ }_{\mid m} \pi^{m j}-\xi^{j}{ }_{\mid m} \pi^{i m} .
\end{aligned}
$$

These boundary conditions are invariant under hypersurface deformations $\left(\xi^{\perp} \equiv \xi, \xi^{i}\right)$ that behave asymptotically as [6]

$$
\begin{gathered}
\xi=b_{i} x^{i}+a(\mathbf{n})+O\left(r^{-1}\right), \\
\xi^{i}=b_{j}^{i} x^{j}+a^{i}(\mathbf{n})+O\left(r^{-1}\right),
\end{gathered}
$$

where $b_{i}$ and $b_{i j}=-b_{j i}$ are arbitrary constants while $a(\mathbf{n})$ and $a^{i}(\mathbf{n})$ are arbitrary functions on the unit sphere $\left(\mathbf{n}^{i}=\frac{x^{i}}{r}\right)$. The constants $b_{i}$ parametrize the Lorentz boosts (the corresponding term $-b^{i} x^{0}$ in $\xi^{i}$ can be absorbed in $a^{i}$ at any given time), whereas the antisymmetric constants $b_{i j}=-b_{j i}$ parametrize the spatial rotations. The zero modes $a_{0}$ and $a_{0}^{i}$ of $a$ and $a^{i}$ are standard translations. General functions $a$ and $a^{i}$ describe "angledependent" translations. The boundary conditions (2.1) and (2.2) are therefore invariant under an asymptotic algebra that has the Poincaré algebra as a subalgebra. We note that with (2.1) and (2.2), the constraints have the following fall-off,

$$
\mathcal{H}=O\left(r^{-3}\right), \quad \mathcal{H}_{i}=O\left(r^{-3}\right)
$$

(in asymptotically Cartesian coordinates).

In addition to containing the Schwarzschild and Kerr solutions and being invariant under (at least) the Poincaré transformations, consistent boundary conditions should fulfill two addition requirements:

- The surface integrals yielding the charges associated with the asymptotic symmetries should be finite and "integrable". By "integrable", one means that the variation of the surface charge, which is a one-form in field space obtained from the bulk generator through integration by parts [6], is exact.

- The kinetic term " $p \ddot{q}$ ", i.e., $\int d^{3} x \pi^{i j} \dot{g}_{i j}$, should be finite, i.e., the symplectic structure should be well-defined.

The general boundary conditions (2.1) and (2.2) fail on both accounts. For that reason, they must be strengthened, but in way that does not eliminate the Schwarzschild or Kerr solutions and keeps the Poincaré transformations among the asymptotic symmetries.

The parity conditions given in [6] fulfill all the consistency requirements. These parity conditions are extra conditions on the leading terms in the expansion (2.1) and (2.2), which are requested to fulfill definite parity properties under the antipodal map $x^{k} \rightarrow-x^{k}$. Explicitly:

$$
\bar{h}_{i j}\left(-n^{k}\right)=\bar{h}_{i j}\left(n^{k}\right), \quad \bar{\pi}^{i j}\left(-n^{k}\right)=-\bar{\pi}^{i j}\left(n^{k}\right) .
$$

These parity conditions are obeyed by the Schwarzchild and Kerr solutions. They are invariant under the transformations (2.7) and (2.8) provided $a-a_{0}$ and $a^{i}-a_{0}^{i}$ are odd 
functions of $\mathbf{n}^{i}$, and thus are in particular invariant under the Poincaré algebra. They play a central role in the mathematical work [50-52].

The parity conditions of [6] make the kinetic term finite since the coefficient of the leading logarithmic singularity in

$$
\int d^{3} x \pi^{i j} \dot{h}_{i j}=\int \frac{d r}{r} \int \sin \theta d \theta d \varphi \bar{\pi}^{i j} \dot{\bar{h}}_{i j}+\cdots
$$

actually vanishes. Indeed, the term $\bar{\pi}^{i j} \dot{\bar{h}}_{i j}$ is an odd function on the sphere, so that its integral over the sphere is zero. The remaining terms in (2.11), denoted by dots, are finite since their integrands decrease strictly faster than $r^{-1}$. The parity conditions also render the Poincaré charges finite and integrable [6]. However, the charges associated with the remaining angle-dependent translations are then found to be identically zero (except the spacetime momentum associated with the zero modes), so that the actual asymptotic symmetry algebra, obtained by taking the quotient of all the asymptotic symmetries by the pure gauge ones - i.e., the ones with zero charges [53] —, is the finite-dimensional Poincaré algebra. There is no room for the full BMS algebra with the parity conditions of [6].

\subsection{Spherical coordinates}

Boundary conditions. It turns out that an alternative strenghtening of the boundary conditions exists, which is also consistent, but which admits the full BMS algebra as asymptotic symmetry algebra. These boundary conditions are based on different parity conditions and do not eliminate solutions with non-vanishing BMS charges.

To describe this alternative strenghtening of the boundary conditions, it is convenient to use spherical coordinates $\left(r, x^{A}\right)$ where $x^{A}$ are coordinates on the sphere. In these coordinates, the asymptotic conditions (2.1) and (2.2) read

$$
\begin{aligned}
g_{r r} & =1+\frac{1}{r} \bar{h}_{r r}+\frac{1}{r^{2}} h_{r r}^{(2)}+o\left(r^{-2}\right), \\
g_{r A} & =\frac{1}{r} h_{r A}^{(2)}+o\left(r^{-1}\right), \\
g_{A B} & =r^{2} \bar{\gamma}_{A B}+r \bar{h}_{A B}+h_{A B}^{(2)}+o(1), \\
\pi^{r r} & =\bar{\pi}^{r r}+\frac{1}{r} \pi^{(2) r r}+o\left(r^{-1}\right), \\
\pi^{r A} & =\frac{1}{r} \bar{\pi}^{r A}+\frac{1}{r^{2}} \pi^{(2) r A}+o\left(r^{-2}\right), \\
\pi^{A B} & =\frac{1}{r^{2}} \bar{\pi}^{A B}+\frac{1}{r^{3}} \pi^{(2) A B}+o\left(r^{-3}\right),
\end{aligned}
$$

where $\bar{\gamma}_{A B}$ is the unit metric on the sphere. There can in fact be $O(1)$-terms $\bar{h}_{r A}$ in the metric coefficients $g_{r A}$ in (2.13), but we have assumed them to vanish. The leading terms in $g_{r A}$ can indeed always be set to zero by a change of coordinates of the form

$$
r^{\prime}=r+o\left(r^{0}\right), \quad x^{\prime A}=x^{A}+\frac{1}{r} \widetilde{X}^{A}\left(x^{B}\right)+o\left(r^{-1}\right) .
$$

The Schwarzchild and Kerr solutions fulfill the condition (2.13), which is preserved under Poincaré transformations (see below). It is only under this condition that we shall develop 
the formalism. Difficulties with integrability of the charges arise when the $O(1)$-terms $\bar{h}_{r A}$ in the metric coefficient $g_{r A}$ do not vanish, but we have not investigated them here since these terms do not appear to carry physical information, at least for the known solutions. A similar stronger-than-expected fall-off of the mixed radial-angular components of the metric was imposed for asymptotically anti-de Sitter spacetimes in [54], or in the hyperbolic description of [48].

It is convenient for later purposes to trade the variable $g_{r r}$ for $\lambda \equiv \frac{1}{\sqrt{g^{r r}}}$, the asymptotic expansion of which is

$$
\lambda=1+\frac{1}{r} \bar{\lambda}+\frac{1}{r^{2}} \lambda^{(2)}+o\left(r^{-2}\right)
$$

with

$$
\bar{\lambda}=\frac{1}{2} \bar{h}_{r r}
$$

Similarly, we introduce

$$
\bar{k}_{B}^{A}=\frac{1}{2} \bar{h}_{B}^{A}+\bar{\lambda} \delta_{B}^{A}, \quad \bar{k}=\bar{k}^{A B} \bar{\gamma}_{A B}
$$

The functions $\bar{k}_{A B}$ on the sphere have the following geometrical meaning. Let $K_{A B}$ be the extrinsic curvature of the 2 -spheres $r=$ constant. If one expands $K_{B}^{A}$ asymptotically, one gets (see appendix A)

$$
K_{B}^{A}=-\frac{1}{r} \delta_{B}^{A}+\frac{1}{r^{2}} \bar{k}_{B}^{A}+\frac{1}{r^{3}} k_{B}^{(2)}{ }_{B}^{A}+o\left(r^{-3}\right),
$$

i.e., $\bar{k}_{B}^{A}$ is the coefficient of the leading perturbation to $K_{B}^{A}$ from its background value $-\frac{1}{r} \delta_{B}^{A}$.

Asymptotic symmetries. In polar coordinates, the transformations that preserve the above boundary conditions have the following behaviour at infinity:

$$
\begin{array}{ll}
\xi=r b+f+O\left(r^{-1}\right), & \xi^{r}=W+O\left(r^{-1}\right), \quad \xi^{A}=Y^{A}+\frac{1}{r} I^{A}+O\left(r^{-2}\right), \\
\bar{D}_{A} \bar{D}_{B} b+\bar{\gamma}_{A B} b=0, & \mathcal{L}_{Y} \bar{\gamma}_{A B}=0,
\end{array}
$$

where $I^{A}$ is given in terms of $b$ and $W$ as

$$
I^{A}=\frac{2 b}{\sqrt{\bar{\gamma}}} \bar{\pi}^{r A}+\bar{D}^{A} W
$$

Here, $b, f, W, Y^{A}$ and $I^{A}$ are functions and vector fields defined on the sphere, and $\bar{D}_{A}$ is the covariant derivative associated with the unit metric $\bar{\gamma}_{A B}$ on the sphere.

A few comments are in order:

- The function $b$ describes the Lorentz boosts. Explicitly, in terms of the cartesian parameters $b_{i}$, one has

$$
b=b_{1} \sin \theta \cos \varphi+b_{2} \sin \theta \sin \varphi+b_{3} \cos \theta,
$$

which is the general solution of $\bar{D}_{A} \bar{D}_{B} b+\bar{\gamma}_{A B} b=0$. 
- The vectors $Y^{A}$ describe the spatial rotations and are the standard Killing vectors on the sphere,

$$
Y=m^{1}\left(-\sin \varphi \frac{\partial}{\partial \theta}-\frac{\cos \theta}{\sin \theta} \cos \varphi \frac{\partial}{\partial \varphi}\right)+m^{2}\left(\cos \varphi \frac{\partial}{\partial \theta}-\frac{\cos \theta}{\sin \theta} \sin \varphi \frac{\partial}{\partial \varphi}\right)+m^{3} \frac{\partial}{\partial \varphi} .
$$

- $f$ contains the time translation through its zero mode $f_{0}(f \equiv a$ in the above parametrization); the other modes define transformations outside the Poincaré algebra.

- $W$ contains the spatial translations. In an expansion in terms of spherical harmonics $Y^{\ell}{ }_{m}$, the translations are the spin-1 part, $W_{P}=\sum_{m=-1}^{1} P^{m} Y_{m}^{1}\left(x^{A}\right)$. One has

$$
\begin{aligned}
& \frac{\partial}{\partial x}=\sin \theta \cos \varphi \frac{\partial}{\partial r}+\frac{1}{r} \cos \theta \cos \varphi \frac{\partial}{\partial \theta}-\frac{1}{r} \frac{\sin \varphi}{\sin \theta} \frac{\partial}{\partial \varphi} \\
& \frac{\partial}{\partial y}=\sin \theta \sin \varphi \frac{\partial}{\partial r}+\frac{1}{r} \cos \theta \sin \varphi \frac{\partial}{\partial \theta}+\frac{1}{r} \frac{\cos \varphi}{\sin \theta} \frac{\partial}{\partial \varphi} \\
& \frac{\partial}{\partial z}=\cos \theta \frac{\partial}{\partial r}-\frac{1}{r} \sin \theta \frac{\partial}{\partial \theta}
\end{aligned}
$$

and one easily sees that the corresponding vectors $I_{P}^{A}$ on the unit sphere fulfill $I_{P}^{A}=\bar{D}^{A} W_{P}$. Furthermore, $\bar{D}^{A} I_{P}^{B}+\bar{D}^{B} I_{P}^{A}+2 W_{P} \bar{\gamma}^{A B}=0$.

- The equation $I^{A}=\frac{2 b}{\sqrt{\bar{\gamma}}} \bar{\pi}^{r A}+\bar{D}^{A} W$ follows from the preservation of the condition $\bar{h}_{r A}=0$ on the leading order of $g_{r A}$. As we have seen, it is fulfilled by the spatial translation Killing vectors of the flat metric, which has indeed $g_{r A}=0$ (to all orders).

Standard parity conditions. In polar coordinates, the parity conditions of [6] read, in terms of coordinates on the unit sphere for which the antipodal map is $x^{A} \rightarrow-x^{A}$,

$$
\bar{h}_{r r} \sim \bar{\pi}^{r A} \sim \bar{h}_{A B}=\text { even, } \quad \bar{\pi}^{r r} \sim \bar{\pi}^{A B}=\text { odd. } \quad(\mathrm{R}-\mathrm{T})
$$

This implies

$$
\bar{\lambda} \sim \bar{k}_{A B}=\text { even. }
$$

In terms of the traditional coordinates $(\theta, \varphi)$ for which the antipodal map is $\theta \rightarrow \pi-\theta$, $\varphi \rightarrow \varphi+\pi$, this is equivalent to

$$
\begin{aligned}
& \bar{h}_{r r} \sim \bar{\pi}^{r \theta} \sim \bar{\pi}^{\theta \varphi} \sim \bar{h}_{\theta \theta} \sim \bar{h}_{\varphi \varphi}=\text { even }, \\
& \bar{\pi}^{r r} \sim \bar{\pi}^{r \varphi} \sim \bar{\pi}^{\theta \theta} \sim \bar{\pi}^{\varphi \varphi} \sim \bar{h}_{\theta \varphi}=\text { odd } .
\end{aligned}
$$

The leading divergence in the kinetic term reads

$$
\int \frac{d r}{r} \int d \theta d \varphi\left(\bar{\pi}^{r r} \dot{\bar{h}}_{r r}+\bar{\pi}^{A B} \dot{\bar{h}}_{A B}\right)
$$

and vanishes with the R-T parity conditions. The surfaces charges are also finite [6]. The transformations that preserve the R-T boundary conditions are

$$
f-f_{0}=\text { odd }, \quad W-W_{P}=\text { even. } \quad(\mathrm{R}-\mathrm{T})
$$

It is because the arbitrary functions occuring in $f$ and $W\left(f-f_{0}\right.$ and $W-W_{P}$, respectively $)$ have parity opposite to that of the translations that they have identically vanishing surface charges and that there is no room for the BMS symmetry with the parity conditions of [6]. 


\section{New boundary conditions}

\subsection{Explicit form}

As we have annouced, there is a different way to achieve finiteness of both the kinetic term and of the surface charges, without making the BMS charges identically zero. This alternative way involves as a key ingredient the imposition of different parity conditions, preserved under surface deformations for which $f$ and $f_{0}$ have same (even) parity, as well as $W$ and $W_{P}$, which are both odd. These alternative boundary conditions are of mixed type, in the sense that spherical and radial projections of the metric have different parities.

To formulate the new conditions in a simple way, we make the change of variables adapted to the description of the extrinsic geometry of the spheres $r=$ constant. That is, we make the change of variables $\bar{h}_{r r}, \bar{h}_{A B} \rightarrow \bar{\lambda}, \bar{k}_{A B}$, extended to the conjugate momenta so as to preserve the kinetic term,

$$
\int d \theta d \varphi\left(\bar{\pi}^{r r} \dot{\bar{h}}_{r r}+\bar{\pi}^{A B} \dot{\bar{h}}_{A B}\right)=\int d \theta d \varphi\left(\bar{p} \dot{\bar{\lambda}}+\pi_{(k)}^{A B} \dot{\bar{k}}_{A B}\right) .
$$

One finds

$$
\begin{array}{ll}
\bar{\lambda}=\frac{1}{2} h_{r r}, & \bar{k}_{A B}=\frac{1}{2} \bar{h}_{A B}+\bar{\lambda} \bar{\gamma}_{A B}, \\
\bar{p}=2\left(\bar{\pi}^{r r}-\bar{\pi}_{A}^{A}\right), & \pi_{(k)}^{A B}=2 \bar{\pi}^{A B} .
\end{array}
$$

The set of parity conditions on the boundary values proposed in this paper are

$$
\bar{\lambda} \sim \bar{\pi}^{A B}=\text { even, } \quad \bar{p} \sim \bar{k}_{A B} \sim \bar{\pi}^{r A}=\text { odd },
$$

or in terms of $(\theta, \varphi)$-components

$$
\begin{gathered}
\bar{\lambda} \sim \bar{\pi}^{r \varphi} \sim \bar{\pi}^{\theta \theta} \sim \bar{\pi}^{\varphi \varphi} \sim \bar{k}_{\theta \varphi}=\text { even }, \\
\bar{p} \sim \bar{\pi}^{r \theta} \sim \bar{\pi}^{\theta \varphi} \sim \bar{k}_{\theta \theta} \sim \bar{k}_{\varphi \varphi}=\text { odd. }
\end{gathered}
$$

Because the variables $(\bar{\lambda}, \bar{p})$ and $\left(\bar{k}_{A B}, 2 \bar{\pi}^{A B}\right)$ in each conjugate pair have opposite parities, the coefficient (3.1) of the divergent piece in the kinetic term vanishes. The parity of $\bar{\pi}^{r A}$ does not matter in this argument since $\bar{h}_{r A}=0$.

The Schwarschild solution obeys the new parity conditions provided one redefines the radial coordinate $r, r \rightarrow r^{\prime}=r\left(1-\frac{m}{r}\right.$ ), which has the effect of making $\bar{k}_{A B}=0$ (and thus odd). The Kerr solution also obeys these parity conditions after the same radial coordinate transformation is made, because the term $\pi^{r \varphi}$ related to the rotations is subleading: its $O\left(r^{-1}\right)$ piece $\bar{\pi}^{r \varphi}$ vanishes and obeys thus trivially both the R-T parity condition (even) or the new ones (odd). The Taub-NUT solution [55], however, has a non-vanishing $\bar{\pi}^{r \varphi}$ which may be taken to be even and to obey therefore the R-T parity conditions [56]. It is excluded by the new boundary conditions. This does not mean that one cannot handle the Taub-NUT solution, but rather that it corresponds to a different sector that has to be treated separately. With the new boundary conditions, the Taub-NUT solution cannot be regarded as a standard asymptotically flat solution as in [56] — something in any case in line with the fact that it has a different topology. 


\subsection{Constraints}

The new parity conditions do insure finiteness of the symplectic form but do not insure by themselves cancelation of the divergent pieces in the boost charges and angular momentum, contrary to the parity conditions of [6]. Therefore, they must be supplemented by further asymptotic restrictions in order to achieve finiteness of the charges. These extra conditions are extremely mild.

With the boundary conditions (2.12)-(2.17), the constraints have the fall-off (2.9), or, in spherical coordinates, $\mathcal{H}=O\left(r^{-1}\right), \mathcal{H}_{r}=O\left(r^{-1}\right), \mathcal{H}_{A}=O(1)$. The strengthening of the boundary conditions is simply that the leading divergences in the constraints should be absent, i.e., one must impose

$$
\mathcal{H}=o\left(r^{-1}\right), \quad \mathcal{H}_{r}=o\left(r^{-1}\right), \quad \mathcal{H}_{A}=o(1) .
$$

Because the constraints transform among themselves under surface deformations, these extra conditions are consistent. Furthermore, they are very mild as announced, since they of course hold on-shell and hence do not remove any solution.

To recapitulate, the complete set of new boundary conditions proposed in this paper is (2.12)-(2.17) with (3.4) and (3.7).

\subsection{Preservation under surface deformations}

The new boundary conditions are invariant under the surface deformations (2.23), (2.24) and (2.25) provided the functions $f$ and $W$ on the sphere fulfill the following conditions:

- The function $f$ has the form

$$
f=-3 b \bar{\lambda}-\frac{1}{2} b \bar{h}+T \equiv-b \bar{\lambda}-b \bar{k}+T,
$$

where $T$ is an arbitrary even function on the sphere,

$$
T=\text { even }
$$

- The function $W$ is an arbitrary odd function on the sphere,

$$
W=\text { odd } .
$$

The transformations that preserve the boundary conditions contain therefore the Poincaré transformations. There are in addition arbitrary angle-dependent translations, but now these have the same parity as the ordinary translations. We shall show in section 4 below that the corresponding charges are all integrable and finite.

The term $-b \bar{\lambda}-b \bar{k}$ must be included in $f$ to cancel terms with incorrect parity in the variation of the canonical variables. For instance, $\delta_{\xi} \bar{\pi}^{r A}$ reads

$$
\delta_{\xi} \bar{\pi}^{r A}=\mathcal{L}_{Y} \bar{\pi}^{r A}+\sqrt{\bar{\gamma}}\left(\bar{D}_{B}\left(b \bar{k}^{B A}\right)-b \bar{D}^{A} \bar{k}-\bar{D}^{A}(\bar{\lambda} b)-\bar{D}^{A} f\right) .
$$


The term $-\bar{D}^{A}(\bar{\lambda} b)$ is odd, rather than being even to conform with the parity of $\bar{\pi}^{r A}$, and must therefore be cancelled by $-\bar{D}^{A} f$. Together with the requirement of integrability of the charges discussed below, this forces $f$ to be given by (3.8).

Under a transformation generated by the gauge parameters (2.23) and (2.24) with $f=T-b \bar{k}-b \bar{\lambda}$ and $I_{A}$ given by (2.25), the components of the metric have the following behaviour

$$
\begin{aligned}
\delta_{\xi} \bar{k}_{A B}= & \mathcal{L}_{Y} \bar{k}_{A B}+\bar{D}_{A} \bar{D}_{B} W+W \bar{\gamma}_{A B} \\
& +\frac{b}{\sqrt{\bar{\gamma}}}\left(\bar{\pi}_{A B}-\bar{\gamma}_{A B} \bar{\pi}\right)+\frac{1}{\sqrt{\bar{\gamma}}} \bar{D}_{A}\left(b \bar{\pi}^{r C} \bar{\gamma}_{C B}\right)+\frac{1}{\sqrt{\bar{\gamma}}} \bar{D}_{B}\left(b \bar{\pi}^{r C} \bar{\gamma}_{C A}\right), \\
\delta_{\xi} \bar{\lambda}= & \frac{b}{4 \sqrt{\bar{\gamma}}} \bar{p}+Y^{C} \partial_{C} \bar{\lambda}
\end{aligned}
$$

where $\bar{\pi}=\bar{\pi}^{A B} \bar{\gamma}_{A B}$. For the momenta, one has

$$
\begin{aligned}
\delta_{\xi} \bar{p}= & \mathcal{L}_{Y} \bar{p}+\sqrt{\bar{\gamma}}\left(4 b \bar{D}_{C} \bar{D}^{C} \bar{\lambda}+4 \bar{D}^{C} b \partial_{C} \bar{\lambda}+12 b \bar{\lambda}\right) \\
\delta_{\xi} \bar{\pi}^{r A}= & \mathcal{L}_{Y} \bar{\pi}^{r A}+\sqrt{\bar{\gamma}}\left(\bar{D}_{B}\left(b \bar{k}^{B A}\right)+\bar{D}^{A} b \bar{k}-\bar{D}^{A} T\right), \\
\delta_{\xi} \bar{\pi}^{A B}= & \mathcal{L}_{Y} \bar{\pi}^{A B}+\sqrt{\bar{\gamma}}\left(\bar{D}^{A} \bar{D}^{B} T-\bar{\gamma}^{A B} \bar{D}_{C} \bar{D}^{C} T\right)+3 b \sqrt{\bar{\gamma}}\left(\bar{k}^{A B}-\bar{\gamma}^{A B} \bar{k}\right) \\
& +\sqrt{\bar{\gamma}} b\left(\bar{\gamma}^{A B} \bar{D}_{C} \bar{D}^{C} \bar{k}^{A} \bar{D}_{C} \bar{D}^{C} \bar{k}^{A B}-\bar{D}_{C} \bar{D}^{A} \bar{k}^{C B}-\bar{D}_{C} \bar{D}^{B} \bar{k}^{C A}\right) \\
& +\sqrt{\bar{\gamma}}\left(-\bar{D}^{A} b \bar{D}^{B} \bar{k}-\bar{D}^{B} b \bar{D}^{A} \bar{k}+\bar{\gamma}^{A B} \bar{D}_{C} b \bar{D}^{C} \bar{k}+2 \bar{\gamma}^{A B} \bar{D}^{D} \bar{k}_{D}^{C} \partial_{C} b\right. \\
& \left.\quad-\bar{D}^{A} \bar{k}^{B C} \partial_{C} b-\bar{D}^{B} \bar{k}^{A C} \partial_{C} b+\bar{D}^{C} \bar{k}^{A B} \partial_{C} b\right) .
\end{aligned}
$$

In order to obtain the transformation law of $\bar{p}$, we used the identity

$$
\bar{D}^{A} \bar{D}^{B} \bar{k}_{A B}-\bar{D}_{A} \bar{D}^{A} \bar{k}=0
$$

coming from the extra condition $\mathcal{H}=o\left(r^{-1}\right)$ (see appendices A and B).

One can verify from these formulas that the parity conditions are all preserved by the surface deformations. Note that for the boosted Schwarzschild metric in the coordinates for which $\bar{k}_{A B}=0$, the only component of the momentum that acquires a non vanishing value is $\bar{p}$, which is correctly odd and equal in this case to $\bar{\pi}^{r r}$.

\section{Asymptotic charges}

We now show that the canonical generators of the asymptotic symmetries are well-defined with the new parity conditions. We follow the method of [6] and do not impose $\bar{h}_{r A}=0$ to begin with. This will be done later, at the point where it is needed. We set $\lambda_{A} \equiv g_{r A}$.

Our aim is to show that the bulk piece of the generators, given by the smeared constraints $\int d^{3} x\left(\xi \mathcal{H}+\xi^{i} \mathcal{H}_{i}\right)$, can be supplemented by appropriate surface terms that make the sum "differentiable" when $\xi$ and $\xi^{i}$ are given by (2.23), (2.24), (2.25) and (3.8), where $T$ and $W$ are arbitrary field-independent even and odd functions, respectively. The boost and rotation parameters $b$ and $Y^{A}$ are also taken to be field independent. 
Taking a general variation of the smeared constraints, we obtain:

$$
\delta \int d^{3} x\left(\xi \mathcal{H}+\xi^{i} \mathcal{H}_{i}\right)=\int d^{3} x\left(\delta_{\xi} \pi^{i j} \delta g_{i j}-\delta_{\xi} g_{i j} \delta \pi^{i j}\right)+\lim _{r \rightarrow \infty} \mathcal{K}_{\xi}\left[\delta g_{i j}, \delta \pi^{i j}\right]
$$

where the boundary term is given by

$$
\begin{aligned}
\mathcal{K}_{\xi}\left[\delta g_{i j}, \delta \pi^{i j}\right]=\oint d^{2} x\{ & -2 \xi^{i} \delta \pi_{i}^{r}+\xi^{r} \pi^{i j} \delta g_{i j}-2 \sqrt{\gamma} \xi \delta K \\
& \left.-\sqrt{\gamma} \gamma^{B C} \delta \gamma_{C A}\left(\xi K_{B}^{A}+\frac{1}{\lambda}\left(\partial_{r} \xi-\lambda^{D} \partial_{D} \xi\right) \delta_{B}^{A}\right)\right\} .
\end{aligned}
$$

In order to write this term, we used a radial $2+1$ split of the $3 \mathrm{~d}$ metric $g_{i j}$ (see appendix $\mathrm{A}$ for more details, including conventions).

Collecting all divergent and finite terms, we get

$$
\begin{aligned}
\mathcal{K}_{\xi}\left[\delta g_{i j}, \delta \pi^{i j}\right]=r \oint d^{2} x\{ & \left.-2 Y^{A} \bar{\gamma}_{A B} \delta \bar{\pi}^{r B}-2 \sqrt{\bar{\gamma}} b \delta \bar{k}\right\} \\
+\oint d^{2} x\{ & -2 Y^{A} \delta\left(\bar{h}_{A B} \bar{\pi}^{r B}+\bar{\gamma}_{A B} \pi^{(2) r B}+\bar{\lambda}_{A} \bar{\pi}^{r r}\right)-2 I^{A} \bar{\gamma}_{A B} \delta \bar{\pi}^{r B} \\
& -2 W \delta \bar{\pi}^{r r}-\sqrt{\bar{\gamma}}\left(b \bar{h} \delta \bar{k}+2 f \delta \bar{k}+2 b \delta k^{(2)}\right) \\
& \left.+\sqrt{\bar{\gamma}}\left(f+\bar{\lambda} b+\bar{\lambda}^{D} \partial_{D} b\right) \delta \bar{h}-\sqrt{\bar{\gamma}} b \bar{k}^{A B} \delta \bar{h}_{A B}\right\}+o\left(r^{0}\right) .
\end{aligned}
$$

If non-zero, the first term is divergent. This is what motivated the introduction of the parity conditions (2.10) in [6], which makes the potentially divergent term identically zero.

Here, a different mechanism is at play. That is, the fact that the constraints hold asymptotically in the sense of (3.7) is sufficient to remove the divergence (independently in fact of any parity condition). In that sense, the parity condition of [6] "kills twice" the divergence. Indeed, the leading terms of the constraints take the form

$$
\mathcal{H}_{A}=-2 \bar{\gamma}_{A B}\left(\bar{\pi}^{r B}+\bar{D}_{C} \bar{\pi}^{B C}\right)+o(1), \quad \mathcal{H}=-\frac{2}{r} \sqrt{\bar{\gamma}}\left(\bar{D}_{A} \bar{D}_{B} \bar{k}^{A B}-\bar{D}_{A} \bar{D}^{A} \bar{k}\right)+o\left(r^{-1}\right)
$$

(see appendix B for more information). Since both these terms are equal to zero by (3.7), we can rewrite the divergent contribution as

$$
\begin{aligned}
\mathcal{K}_{\xi}\left[\delta g_{i j}, \delta \pi^{i j}\right]=r \oint d^{2} x & \left\{2 Y^{A} \bar{\gamma}_{A B} \bar{D}_{C} \delta \bar{\pi}^{B C}\right. \\
& \left.-2 \sqrt{\bar{\gamma}} b \delta\left(\bar{k}-\bar{D}_{A} \bar{D}_{B} \bar{k}^{A B}+\bar{D}_{A} \bar{D}^{A} \bar{k}\right)\right\}+O(1) .
\end{aligned}
$$

If we then integrate by parts and use the properties of the Lorentz parameters given in (2.24), we see that this divergence cancels. 
Using the fact that both $Y^{A}$ and $b$ are field independent, we can partially integrate the finite part of the boundary term:

$$
\begin{aligned}
& \mathcal{K}_{\xi}\left[\delta g_{i j}, \delta \pi^{i j}\right]=\delta \oint d^{2} x\left\{-2 Y^{A}\left(\bar{h}_{A B} \bar{\pi}^{r B}+\bar{\gamma}_{A B} \pi^{(2) r B}+\bar{\lambda}_{A} \bar{\pi}^{r r}\right)\right. \\
&\left.-2 \sqrt{\bar{\gamma}} b k^{(2)}-\sqrt{\bar{\gamma}} \frac{1}{4} b\left(\bar{h}^{2}+\bar{h}^{A B} \bar{h}_{A B}\right)\right\} \\
&+\oint d^{2} x\left\{-2 I^{A} \bar{\gamma}_{A B} \delta \bar{\pi}^{r B}-2 W \delta \bar{\pi}^{r r}-\sqrt{\bar{\gamma}}(2 f+\bar{h} b) \delta\left(2 \bar{\lambda}+\bar{D}_{A} \bar{\lambda}^{A}\right)\right. \\
&\left.+\sqrt{\bar{\gamma}}\left(\bar{\lambda}^{C} \partial_{C} b \bar{\gamma}^{A B}-b \bar{D}^{A} \bar{\lambda}^{B}\right) \delta \bar{h}_{A B}\right\}+o\left(r^{0}\right) .
\end{aligned}
$$

To integrate the terms written in the last line, we now use the conditions $\bar{\lambda}_{A}=0$ and (3.8). Without restriction on these parameters, the one-form in field space $\mathcal{K}_{\xi}$ is not exact. ${ }^{1}$ We then get

$$
\mathcal{K}_{\xi}\left[\delta g_{i j}, \delta \pi^{i j}\right]=-\delta \mathcal{B}_{\xi}\left[g_{i j}, \pi^{i j}\right]
$$

where

$$
\begin{aligned}
\mathcal{B}_{\xi}\left[g_{i j}, \pi^{i j}\right]= & \oint d^{2} x\left\{Y^{A}\left(4 \bar{k}_{A B} \bar{\pi}^{r B}-4 \bar{\lambda} \bar{\gamma}_{A B} \bar{\pi}^{r B}+2 \bar{\gamma}_{A B} \pi^{(2) r B}\right)+W\left(2 \bar{\pi}^{r r}-2 \bar{D}_{A} \bar{\pi}^{r A}\right)\right. \\
& \left.+T 4 \sqrt{\bar{\gamma}} \bar{\lambda}+b \sqrt{\bar{\gamma}}\left(2 k^{(2)}+\bar{k}^{2}+\bar{k}_{B}^{A} \bar{k}_{A}^{B}-6 \overline{\lambda k}\right)+b \frac{2}{\sqrt{\bar{\gamma}}} \bar{\gamma}_{A B} \bar{\pi}^{r A} \bar{\pi}^{r B}\right\} .
\end{aligned}
$$

Using the parity conditions (3.4), this boundary term can be simplified to

$\mathcal{B}_{\xi}\left[g_{i j}, \pi^{i j}\right]=\oint d^{2} x\left\{T 4 \sqrt{\bar{\gamma}} \bar{\lambda}+W \bar{p}+Y^{A} 2 \bar{\gamma}_{A B}\left(\pi^{(2) r B}-2 \bar{\lambda}^{r B}\right)+b 2 \sqrt{\bar{\gamma}}\left(k^{(2)}-3 \overline{\lambda k}\right)\right\}$.

It is interesting to note that integrability alone of the term $\sqrt{\bar{\gamma}}(2 f+\bar{h} b) \delta(2 \bar{\lambda})$ leaves some freedom in the choice of $f$, since any function of $\bar{\lambda}$ could be added to $f$ without destroying integrability. On the other hand, the preservation under surface deformations of the parity conditions controls, as we have seen, the $\bar{\lambda}$-dependence and removes the ambiguity. It is very satisfying to see that both the integrability conditions and the parity conditions combine to fix the form of the charges.

We can summarize the above results in the following theorem:

Theorem 4.1 The transformations associated with the asymptotic symmetries

$$
\begin{aligned}
\xi & =b(r-\bar{\lambda}-\bar{k})+T+O\left(r^{-1}\right), & & \xi^{A}=Y^{A}+\frac{1}{r}\left(\bar{D}^{A} W+\frac{2 b}{\sqrt{\bar{\gamma}}} \bar{\pi}^{r A}\right)+O\left(r^{-2}\right), \\
\xi^{r} & =W+O\left(r^{-1}\right), & & \bar{D}_{A} \bar{D}_{B} b+\bar{\gamma}_{A B} b=0, \quad \mathcal{L}_{Y} \bar{\gamma}_{A B}=0, \\
T & =\text { even function }, & & W=\text { odd function },
\end{aligned}
$$

where $b, Y^{A}, T$ and $W$ are field independent, are canonical transformations generated by

$$
G_{\xi}\left[g_{i j}, \pi^{i j}\right]=\int d^{3} x\left(\xi \mathcal{H}+\xi^{i} \mathcal{H}_{i}\right)+\mathcal{B}_{\xi}\left[g_{i j}, \pi^{i j}\right],
$$

where the boundary term $\mathcal{B}_{\xi}$ is given in equation (4.9).

This shows that the asymptotic symmetries have well-defined (differentiable) generators.

\footnotetext{
${ }^{1} \mathrm{~A}$ different viewpoint on integrability has been recently developed in [57].
} 
We close this section with four comments.

- As we pointed out, finiteness of the charges holds even without any parity condition and is a consequence of the asymptotic implementation of the constraints. However, if one drops the parity conditions, there is some ambiguity in the $\bar{\lambda}$-dependence of the boost generators (in addition to the singularity of the symplectic structure).

- Time and spatial translations, respectively given by $T=1$ and $W_{P}=\sum_{m=-1}^{1} P^{m} Y_{1 m}\left(x^{A}\right)$, have associated charges

$$
\mathcal{M}\left[g_{i j}, \pi^{i j}\right]=4 \oint d^{2} x \sqrt{\bar{\gamma}} \bar{\lambda}, \quad \mathcal{P}\left[g_{i j}, \pi^{i j}\right]=\sum_{m=-1}^{1} P^{m} \oint d^{2} x Y_{1 m} \bar{p},
$$

where $\mathcal{M}$ is the mass while $\mathcal{P}$ the linear momentum. These expressions agree with the ADM ones, as they should.

- The situation is more subtle for the Lorentz transformations parametrized by $b$ and $Y^{A}$. Their charges will in general contain non-linear terms in the dynamical fields:

$$
\begin{aligned}
\mathcal{B}_{Y}\left[g_{i j}, \pi^{i j}\right] & =\oint d^{2} x Y^{A} 2 \bar{\gamma}_{A B}\left(\pi^{(2) r B}-2 \bar{\lambda} \bar{\pi}^{r B}\right), \\
\mathcal{B}_{b}\left[g_{i j}, \pi^{i j}\right] & =\oint d^{2} x b 2 \sqrt{\bar{\gamma}}\left(k^{(2)}-3 \overline{\lambda k}\right) .
\end{aligned}
$$

When one uses the R-T parity conditions given in equation (2.10) in the general expression (4.8), all non-linear terms disappear and we recover the results of [6]. But with the new parity conditions, some of the non-linear terms are generically non-vanishing. This non-linearity is similar to what was encountered for anti-de Sitter gravity coupled to a scalar field, either in the holographic renormalization approach [58, 59], or through canonical methods [60-62].

- The charges associated with supertranslations are

$$
\mathcal{B}_{\xi}\left[g_{i j}, \pi^{i j}\right]=\oint d^{2} x\{T 4 \sqrt{\bar{\gamma}} \bar{\lambda}+W \bar{p}\}
$$

and do not vanish in general since they are given by the integrals of non trivial even functions.

\section{The BMS algebra}

In order to compute the algebra of the asymptotic symmetries, we have to take into account the fact that they have an explicit phase-space dependence. The resulting bracket between two asymptotic transformations $\xi_{1}\left(Y_{1}, b_{1}, T_{1}, W_{1}\right)$ and $\xi_{2}\left(Y_{2}, b_{2}, T_{2}, W_{2}\right)$ is then given by

$$
\left[\xi_{1}, \xi_{2}\right]_{M}=\left[\xi_{1}, \xi_{2}\right]_{S D}+\delta_{2}^{g, \pi} \xi_{1}-\delta_{1}^{g, \pi} \xi_{2}+\Theta^{A}\left(\xi_{1}, \xi_{2}\right) \mathcal{H}_{A}+\Theta\left(\xi_{1}, \xi_{2}\right) \mathcal{H}
$$

where $[,]_{S D}$ is the usual surface deformation bracket while the variations $\delta^{g, \pi}$ only hit the explicit dependence on the gravitational fields. The extra terms proportional to the constraints contain the contribution to the Poisson bracket produced when the Euler-Lagrange 
derivatives only hit the gauge parameters. In this case, they can be ignored safely. One way to see this is that the improper part of the gauge parameters explicitly depends only on one element from each canonical pair namely $g_{r r}, \pi^{r A}$ and $g_{A B}$. This implies that non-zero $\Theta$ or $\Theta^{A}$ will always involve the proper part of at least one of the two gauge parameters. As the bracket of a proper gauge transformation with any allowed transformation is a proper gauge transformation, these contributions are always sub-leading.

Using results for the variations of the asymptotic fields given in section 3 , the computation of the algebra of asymptotic transformations is straightforward:

$$
\widehat{\xi}(\widehat{Y}, \widehat{b}, \widehat{T}, \widehat{W})=\left[\xi_{1}\left(Y_{1}, b_{1}, T_{1}, W_{1}\right), \xi_{2}\left(Y_{2}, b_{2}, T_{2}, W_{2}\right)\right]_{M},
$$

where

$$
\begin{aligned}
\widehat{Y}^{A} & =Y_{1}^{B} \partial_{B} Y_{2}^{A}+\bar{\gamma}^{A B} b_{1} \partial_{B} b_{2}-(1 \leftrightarrow 2), \\
\widehat{b} & =Y_{1}^{B} \partial_{B} b_{2}-(1 \leftrightarrow 2), \\
\widehat{T} & =Y_{1}^{A} \partial_{A} T_{2}-3 b_{1} W_{2}-\partial_{A} b_{1} \bar{D}^{A} W_{2}-b_{1} \bar{D}_{A} \bar{D}^{A} W_{2}-(1 \leftrightarrow 2), \\
\widehat{W} & =Y_{1}^{A} \partial_{A} W_{2}-b_{1} T_{2}-(1 \leftrightarrow 2) .
\end{aligned}
$$

When the functions $T$ and $W$ are restricted to be respectively an even and an odd function on the sphere, the bracket obtained here describes the BMS algebra using an unfamiliar basis. The proof can be found in appendix C. The main idea is to relate the algebra obtained here with the asymptotic analysis performed in [49] in the context of the hyperbolic treatment of spatial infinity developed in [70-72]. The result is a linear isomorphism between the set of pairs of functions $(T, W)$ with even and odd parity and the set of functions $\mathcal{T}$ of no definite parity such that $\mathcal{T}$ transforms as a usual BMS supertranslation under the action of Lorentz algebra:

$$
\delta_{Y, b} \mathcal{T}=Y^{A} \partial_{A} \mathcal{T}-\bar{D}^{A} b \partial_{A} \mathcal{T}-b \mathcal{T}
$$

Developing the various functions in spherical harmonics, one can write the first few terms of the change of basis:

$$
\begin{aligned}
W & =\sum_{k} \sum_{m=-2 k-1}^{2 k+1} W_{2 k+1, m} Y_{2 k+1, m}, \quad T=\sum_{k} \sum_{m=-2 k}^{2 k} T_{2 k, m} Y_{2 k, m}, \\
\mathcal{T} & =T_{0,0} Y_{0,0}+\sum_{m=-1}^{1} W_{1, m} Y_{1, m}+\frac{1}{4} \sum_{m=-2}^{2} T_{2, m} Y_{2, m}+\ldots
\end{aligned}
$$

where the dots denotes terms containing spherical harmonics with $l>2$.

General theorems guarantee that the generators associated to asymptotic symmetries close in the Poisson bracket according to the same algebra, possibly modified by central charges [63]. One can check that in the present case, however, the algebra does not acquire a central extension:

$$
\left\{G_{\xi_{1}}\left[g_{i j}, \pi^{i j}\right], G_{\xi_{2}}\left[g_{i j}, \pi^{i j}\right]\right\}=G_{\widehat{\xi}}\left[g_{i j}, \pi^{i j}\right]
$$

The easiest way to check this is to express the Poisson bracket as a variation $\left\{G_{\xi_{1}}, G_{\xi_{2}}\right\}=\delta_{\xi_{2}} G_{\xi_{1}}$ and evaluate the result on the background Minkowski space. 


\section{Conclusions}

In this paper, we have proposed new boundary conditions for asymptotically flat spacetimes at spatial infinity. These new boundary are given by (2.12)-(2.17), with the parity conditions (3.4) on the leading order of the asymptotic fields and the requested constraint fall-off (3.7).

These boundary conditions fulfill all the standard consistency requirements: they contain the Schwarzschild and Kerr solutions as well as their Poincaré transforms; the symplectic structure is well-defined; the generators of the asymptotic symmetries, which contain asymptotic Poincaré transformations, are all finite. We have also constructed explicitly the conserved charges of the asymptotic symmetries and showed that they close according to the BMS algebra, which is consequently the asymptotic symmetry algebra.

We have therefore achieved the goal outlined in the introduction, of associating standard canonical generators at spatial infinity to the BMS symmetry transformations first revealed at null infinity. These generators do not identically vanish and hence have a non trivial action in the physical phase space.

The new boundary conditions reduce the number of arbitrary functions of the angles $a(\mathbf{n})$ and $a^{i}(\mathbf{n})$ appearing in (2.7) and (2.8) - namely 4 - to a single function of the angles, in agreement with the BMS symmetry. Crucial in this reduction are the conditions $\bar{h}_{r A}=0$, which are imposed in our approach in order to guarantee integrability of the charges.

Another crucial ingredient are the parity conditions (3.4), which are different from those proposed earlier in [6]. We have seen that the BMS super-translations are encoded in the odd part of $W$ and the even part of $T$. Both of these parts are incompatible with the parity conditions of [6] and hence absent in that approach, except for the few spherical harmonics describing Poincaré translations. [The even part of $W$ and the odd part of $T$ are compatible with the boundary conditions of [6] but have zero charges because their parity is opposite to that of the Poincaré translations. Hence they are pure gauge.]

By contrast, our new parity conditions allow arbitrary odd $W$ 's and even T's and a non trivial action of the BMS group at spatial infinity. Furthermore, the BMS group at spatial infinity is the same as the BMS group encountered at null infinity, as shown in [49] using the methods developed in [64-66] (see also appendix C).

Our work can be extended in various directions.

- The superrotations [16-20, 22] are not included among the asymptotic symmetries described here. It would be of interest to examine if and how they can be covered.

- The new parity conditions do not include the Taub-NUT solution. To cover it, one presumably needs to consider it as defining a distinct "sector" and study perturbations around it ("asymptotically Taub-NUT spacetimes"). It would be useful to carry out the study explicitly. This would need a more detailed analysis of the electric and magnetic components of the Weyl tensor at infinity.

- Our paper focused on vacuum gravity. Matter fields, and most notably, the electromagnetic field, should be included. Concerning the latter, a first step has been done 
in [67] where the authors have realized at spatial infinity, using a hyperbolic slicing, a description of the enlarged asymptotic symmetry of electromagnetism introduced in $[68,69]$.

- Finally, it would be of interest to investigate possible relaxations of the boundary conditions beyond parity conditions [48]. We have seen that the asymptotic implementation of the constraints, without parity conditions, is sufficient to ensure finiteness of the charges but not of the canonical kinetic term $\int d t p \dot{q}$. The authors of [48] use the framework of holographic renormalisation to remove the divergences that appear in the symplectic structure but end up with a puzzle that they lucidly describe in their conclusions: instead of a single phase space, they get a collection of phase spaces where some BMS transformations are not allowed to act. How this would translate in the ADM approach is worth pursuing.

In a related context, a different set of boundary conditions at spatial infinity having a BMS algebra as symmetry has been presented in [73]. However, this representation of the BMS algebra does not contain spatial translations nor Lorentz boosts and, as such, is not the usual BMS algebra considered at null infinity. Their analysis is nevertheless very interesting and it may hint at possible generalisations of the results presented in this work.

It is hoped to return to these questions in the future.

\section{Acknowledgments}

This work was partially supported by the ERC Advanced Grant "High-Spin-Grav", by FNRS-Belgium (convention FRFC PDR T.1025.14 and convention IISN 4.4503.15) and by the "Communauté Française de Belgique" through the ARC program.

\section{A Radial decomposition of the spatial metric and the spatial curvature}

Let us assume that we have spatial coordinates given by $x^{i}=\left(r, x^{A}\right)$ where $x^{A}$ are coordinates on the 2-sphere. We introduce:

$$
\gamma_{A B} \equiv g_{A B}, \quad \lambda_{A} \equiv g_{r A}, \quad \lambda \equiv \frac{1}{\sqrt{g^{r r}}} .
$$

The metric and its inverse take the form:

$$
g_{i j}=\left(\begin{array}{cc}
\lambda^{2}+\lambda_{C} \lambda^{C} & \lambda_{B} \\
\lambda_{A} & \gamma_{A B}
\end{array}\right), \quad g^{i j}=\left(\begin{array}{cc}
\frac{1}{\lambda^{2}} & -\frac{\lambda^{B}}{\lambda^{2}} \\
-\frac{\lambda^{A}}{\lambda^{2}} & \gamma^{A B}+\frac{\lambda^{A} \lambda^{B}}{\lambda^{2}}
\end{array}\right),
$$

where we used $\gamma_{A B}$ and its inverse $\gamma^{A B}$ to raise and lower the angular indices $A, B, \ldots$ 
Introducing the extrinsic curvature of the 2 -spheres $K_{A B}$, we can write all the Christoffel symbols:

$$
\begin{aligned}
K_{A B}= & \frac{1}{2 \lambda}\left(-\partial_{r} g_{A B}+D_{A} \lambda_{B}+D_{B} \lambda_{A}\right), \\
\Gamma_{A B}^{r}= & \frac{1}{\lambda} K_{A B}, \\
\Gamma_{B C}^{A}= & { }^{\gamma} \Gamma_{B C}^{A}-\frac{\lambda^{A}}{\lambda} K_{B C}, \\
\Gamma_{r A}^{r}= & \frac{1}{\lambda}\left(\partial_{A} \lambda+K_{A B} \lambda^{B}\right), \\
\Gamma_{r r}^{r}= & \frac{1}{\lambda} \partial_{r} \lambda+\frac{\lambda^{A}}{\lambda}\left(\partial_{A} \lambda+K_{A B} \lambda^{B}\right), \\
\Gamma_{r B}^{A}= & -\frac{\lambda^{A}}{\lambda}\left(\partial_{B} \lambda+K_{B C} \lambda^{C}\right)+D_{B} \lambda^{A}-\lambda K_{B}^{A}, \\
\Gamma_{r r}^{A}= & -\lambda\left(\gamma^{A B}+\frac{\lambda^{A} \lambda^{B}}{\lambda^{2}}\right)\left(\partial_{B} \lambda+K_{B C} \lambda^{C}\right)-\lambda^{C}\left(D^{A} \lambda_{C}-\lambda K_{C}^{A}\right) \\
& \quad-\frac{\lambda^{A}}{\lambda} \partial_{r} \lambda+\gamma^{A B} \partial_{r} \lambda_{B},
\end{aligned}
$$

where $D_{A}$ is the covariant derivative associated to $\gamma_{A B}$.

The Ricci tensor is given by:

$$
\begin{aligned}
{ }^{(3)} R_{A B}= & \frac{1}{\lambda} \partial_{r} K_{A B}+2 K_{A C} K_{B}^{C}-K K_{A B}-\frac{1}{\lambda} D_{A} D_{B} \lambda \\
& +{ }^{\gamma} R_{A B}-\frac{1}{\lambda} \mathcal{L}_{\lambda} K_{A B}, \\
{ }^{(3)} R_{r A}= & \lambda\left(\partial_{A} K-D_{B} K_{A}^{B}\right)+{ }^{(3)} R_{A B} \lambda^{B}, \\
{ }^{(3)} R_{r r}= & \lambda\left(\partial_{r} K-\lambda^{A} \partial_{A} K\right)-\lambda^{2} K_{B}^{A} K_{A}^{B}-\lambda D_{A} D^{A} \lambda \\
& -{ }^{(3)} R_{A B} \lambda^{A} \lambda^{B}+2^{(3)} R_{r B} \lambda^{B},
\end{aligned}
$$

while the Ricci scalar takes the form

$$
{ }^{(3)} R=\frac{2}{\lambda}\left(\partial_{r} K-\lambda^{A} \partial_{A} K\right)+{ }^{\gamma} R-K_{B}^{A} K_{A}^{B}-K^{2}-\frac{2}{\lambda} D_{A} D^{A} \lambda .
$$

The asymptotic conditions considered in section 2 imply

$$
\begin{aligned}
\lambda & =1+\frac{1}{r} \bar{\lambda}+\frac{1}{r^{2}} \lambda^{(2)}+o\left(r^{-2}\right), \quad \lambda^{A}=\frac{1}{r^{2}} \bar{\lambda}^{A}+\frac{1}{r^{3}} \lambda^{(2) A}+o\left(r^{-3}\right), \\
K_{B}^{A} & =-\frac{1}{r} \delta_{B}^{A}+\frac{1}{r^{2}} \bar{k}_{B}^{A}+\frac{1}{r^{3}} k_{B}^{(2)}{ }_{B}^{A}+o\left(r^{-3}\right),
\end{aligned}
$$

where

$$
\bar{\lambda}=\frac{1}{2} \bar{h}_{r r}, \quad \bar{\lambda}^{A}=\bar{h}_{r}^{A}=\bar{h}_{r B} \bar{\gamma}^{B A}, \quad \bar{k}_{B}^{A}=\frac{1}{2} \bar{h}_{B}^{A}+\bar{\lambda} \delta_{B}^{A}+\frac{1}{2} \bar{D}^{A} \bar{\lambda}_{B}+\frac{1}{2} \bar{D}_{B} \bar{\lambda}^{A} .
$$

The indices on the barred quantities are lowered and raised with $\bar{\gamma}_{A B}$ and its inverse $\bar{\gamma}^{A B}$. 


\section{B More details on the divergences of Lorentz generators}

In this appendix two things are done.

- First, we give the first two leading orders in the asymptotic expansion of the constraints.

- Second, we clarify the appearance (and non-appearance) of logarithmic terms in the asymptotic expansion of the fields. Some explicit examples of solutions to the gravitational constraints equations with logarithmically divergent Lorentz charges have been constructed [51]. We make the link with this analysis.

As before, we assume $\bar{\lambda}^{A}=0$.

Expending the momentum constraint $\mathcal{H}_{A}$ to second order, we get

$$
\begin{aligned}
\mathcal{H}_{A}= & -2\left(\partial_{r} \pi_{A}^{r}+\partial_{B} \pi_{A}^{B}-{ }^{\gamma} \Gamma_{B A}^{C} \pi_{C}^{B}-\frac{1}{\lambda} \partial_{A} \lambda \pi_{r}^{r}\right)+o\left(r^{-1}\right) \\
= & -2 \bar{\gamma}_{A B}\left(\bar{\pi}^{r B}+\bar{D}_{C} \bar{\pi}^{B C}\right) \\
& -\frac{2}{r}\left[\bar{\gamma}_{A B} \bar{D}_{C} \pi^{(2) B C}-\partial_{A} \bar{\lambda} \bar{\pi}^{r r}+\bar{D}_{B}\left(\bar{h}_{A C} \bar{\pi}^{B C}\right)-\frac{1}{2} \bar{\pi}^{B C} \bar{D}_{A} \bar{h}_{C B}\right]+o\left(r^{-1}\right),
\end{aligned}
$$

which, in particular, implies

$$
\bar{\gamma}_{A B} \bar{D}_{C}\left(\pi^{(2) B C}-2 \bar{\lambda} \bar{\pi}^{B C}\right)=\partial_{A} \bar{\lambda}\left(\bar{\pi}^{r r}-\bar{\pi}_{B}^{B}\right)-2 \bar{D}_{B}\left(\bar{k}_{A C} \bar{\pi}^{B C}\right)+\bar{\pi}^{B C} \bar{D}_{A} \bar{k}_{C B} .
$$

If we contract the second line with Killing vectors of the sphere $Y^{A}$ and integrate, we obtain three integrability conditions:

$$
\oint d^{2} x\left(\left(\bar{\pi}^{r r}-\bar{\pi}_{B}^{B}\right) Y^{A} \partial_{A} \bar{\lambda}+\bar{\pi}^{B C} \mathcal{L}_{Y} \bar{k}_{B C}\right)=0 .
$$

These conditions are necessary and sufficient to guaranty the existence of $\pi^{(2) A B}$ such that (B.3) is valid. They are the hamiltonian equivalent of the integrability conditions necessary for the existence of solutions at second order in the hyperbolic description as described in [48, 72] (see also [47]).

These conditions are consequences of the asymptotic conditions we imposed on our fields. Looking at (B.1), we see that if the integrability conditions are not satisfied, they produce a logarithmic term in $\pi^{r A}$. In other words, if we assume,

$$
\pi^{r A}=r^{-1} \bar{\pi}^{r A}+\log r r^{-2} \pi^{(l) r A}+r^{-2} \pi^{(2) r A}+o\left(r^{-2}\right),
$$

then equation (B.3) becomes

$$
\begin{aligned}
\bar{\gamma}_{A B} \pi^{(l) r B}= & -\bar{\gamma}_{A B} \bar{D}_{C}\left(\pi^{(2) B C}-2 \bar{\lambda} \bar{\pi}^{B C}\right) \\
& +\partial_{A} \bar{\lambda}\left(\bar{\pi}^{r r}-\bar{\pi}_{B}^{B}\right)-2 \bar{D}_{B}\left(\bar{k}_{A C} \bar{\pi}^{B C}\right)+\bar{\pi}^{B C} \bar{D}_{A} \bar{k}_{C B} .
\end{aligned}
$$

In this case, there is no integrability condition as the logarithmic term will absorb the corresponding contribution. This logarithmic term will then appear in the angular momentum charges as a divergent term. 
The solution described in [51] has the following asymptotic behaviour:

$$
\begin{aligned}
& \bar{\pi}^{r r}=\sqrt{\bar{\gamma}}\left(\beta\left(x^{A}\right)-B_{m} Y_{1, m}\left(x^{A}\right)\right), \quad \bar{\pi}^{r A}=-\sqrt{\bar{\gamma}} \bar{D}^{A}\left(B_{m} Y_{1, m}\left(x^{A}\right)\right), \\
& \bar{\pi}^{A B}=\sqrt{\bar{\gamma} \gamma}{ }^{A B} B_{m} Y_{1, m}\left(x^{A}\right), \quad \bar{\lambda}=\frac{1}{2} A+\frac{1}{4} \alpha\left(x^{A}\right), \quad \bar{k}_{A B}=A \bar{\gamma}_{A B},
\end{aligned}
$$

where $A, B_{m}$ are constants $(m=-1,0,1)$ and $Y_{1, m}$ are $l=1$ spherical harmonics. In this case, the integrability condition is

$$
\left.\oint d^{2} \Omega\left(\beta-3 B_{m} Y_{1, m}\right)\right) Y^{A} \partial_{A} \alpha=0
$$

The solution given in corollary 3.4 of [51] corresponds to a specific choice of $\beta$ and $\alpha$ for which this condition is not fulfilled. In that case, the logarithmic term in the expansion of $\pi^{r A}$ has to be non-zero which will introduce a logarithmic divergence in the angular momentum charges. One can show that this divergence will be exactly given by the value of the integrability condition that reproduces the results of [51].

A similar analysis has to be done for the hamiltonian constraint $\mathcal{H}$. After some algebra, we get

$$
\begin{aligned}
& \mathcal{H}=-\left(1+3 \frac{\bar{\lambda}}{r}\right) \frac{2}{r^{3}} \sqrt{\gamma}\left(\bar{D}_{A} \bar{D}_{B} \bar{k}^{A B}-\bar{D}_{A} \bar{D}^{A} \bar{k}\right) \\
& +\frac{1}{r^{2}}\left\{-\sqrt{\bar{\gamma}}\left[\bar{D}_{A} \bar{D}_{B}\left(h^{(2) A B}+2 \lambda^{(2)} \bar{\gamma}^{A B}+4 \bar{\lambda} k^{A B}\right)\right.\right. \\
& \left.-\bar{D}_{A} \bar{D}^{A}\left(h^{(2)}+4 \lambda^{(2)}+4 \bar{\lambda} k\right)-\left(h^{(2)}+4 \lambda^{(2)}+4 \bar{\lambda} k\right)\right] \\
& -\sqrt{\bar{\gamma}}\left[3 \bar{k}_{B}^{A} \bar{k}_{A}^{B}+4 \bar{k}^{A B} \bar{D}_{C} \bar{D}^{C} \bar{k}_{A B}+4 \bar{k}^{A B} \bar{D}_{A} \bar{D}_{B} \bar{k}-4 \bar{k}^{A B}\left(\bar{D}_{C} \bar{D}_{A}+\bar{D}_{A} \bar{D}_{C}\right) \bar{k}_{A}^{C}\right. \\
& +3 \bar{D}_{A} \bar{k}_{B C} \bar{D}^{A} \bar{k}^{B C}-\bar{D}_{A} k \bar{D}^{A} k-2 \bar{D}_{A} \bar{k}_{B C} \bar{D}^{B} \bar{k}^{A C} \\
& \left.+4 \bar{D}_{B} \bar{k}_{A}^{B}\left(\bar{D}^{A} \bar{k}-\bar{D}_{C} \bar{k}^{C A}\right)-\bar{k}^{2}+12 \bar{\lambda}^{2}+6 \bar{\lambda}_{C} \bar{D}^{C} \bar{\lambda}+4 \bar{D}_{A} \overline{\lambda D}^{A} \bar{\lambda}\right] \\
& \left.+\frac{1}{\sqrt{\bar{\gamma}}}\left[2 \bar{\pi}^{r A} \bar{\pi}_{A}^{r}+\bar{\pi}^{A B} \bar{\pi}_{A B}+\frac{1}{2}\left(\bar{\pi}^{r r}-\bar{\pi}_{A}^{A}\right)^{2}-\left(\bar{\pi}_{A}^{A}\right)^{2}\right]\right\}+o\left(r^{-2}\right) .
\end{aligned}
$$

The sub-leading contribution takes the form

$$
\sqrt{\bar{\gamma}}\left(\bar{D}_{A} \bar{D}_{B} \alpha^{A B}-\bar{D}_{A} \bar{D}^{A} \alpha-\alpha\right)=J,
$$

where $\alpha^{A B}$ is linear in the second-order perturbations of the fields, and $J$ quadratic in their first-order perturbations. Integrating this equation with a "boost" parameter $b$ such that $\bar{D}_{A} \bar{D}_{B} b+\bar{\gamma}_{A B} b=0$, we get the three integrability conditions:

$$
\oint d^{2} x b J=0
$$

on the first-order perturbation. As before, one can show that if $J$ satisfies these identities, then there exists a $h^{(2)}$ such that the constraint is valid. If they do not hold, then logarithmic terms will appear in the expansion of $g_{i j}$. 
The same analysis on the radial constraint $\mathcal{H}_{r}$ does not give any new integrability conditions. It takes the form

$$
\begin{aligned}
\mathcal{H}_{r}= & -\frac{2}{r}\left(\partial_{A} \bar{\pi}^{A r}-\bar{\pi}_{A}^{A}\right)-\frac{2}{r^{2}}\left(-\pi^{(2) r r}-\pi_{A}^{(2)}{ }_{A}^{A}\right. \\
& \left.-\bar{\lambda}\left(\bar{\pi}^{r r}-\bar{\pi}_{A}^{A}\right)-\bar{k}_{A B} \bar{\pi}^{A B}+\partial_{A}\left(\pi^{(2) A r}+2 \bar{\lambda} \bar{\pi}^{A r}\right)\right)+O\left(r^{-3}\right),
\end{aligned}
$$

which imposes

$$
\bar{\pi}_{A}^{A}=\partial_{A} \bar{\pi}^{A r} \quad \Rightarrow \quad \bar{D}_{A} \bar{D}_{B} \bar{\pi}^{A B}+\bar{\pi}_{A}^{A}=0,
$$

and fixes $\pi^{(2) r r}-\pi_{A}^{(2) A}$ in terms of the other asymptotic fields.

The six integrability conditions (B.9), (B.12) for the existence of the subleading terms without the need to introduce logarithms are easily verified to be fulfilled by

- spacetimes satisfying the R-T parity conditions [6]:

$$
\bar{\lambda} \sim \bar{\pi}^{r A} \sim \bar{k}_{A B} \sim \text { even }, \quad \bar{\pi}^{r r} \sim \bar{\pi}^{A B}=\text { odd },
$$

- spacetimes satisfying the new parity conditions:

$$
\bar{\lambda} \sim \bar{\pi}^{A B} \sim \text { even }, \quad\left(\bar{\pi}^{r r}-\bar{\pi}_{A}^{A}\right) \sim \bar{k}_{A B} \sim \bar{\pi}^{r A}=\text { odd } .
$$

The parity conditions guarantee therefore the consistency of the perturbative expansion adopted here.

\section{BMS algebra in the "instant form" and in the hyperbolic form of the dynamics}

We relate in this appendix the results of section 5 to the recent work on the asymptotic symmetry algebra carried out in [49] in the context of the hyperbolic treatment of spatial infinity [70-72], with a Hamiltonian that generates boosts asymptotically. The results of [49] apply to the analysis of [48], which adopts different boundary conditions than the ones taken here (logarithmic terms, no parity condition) and regularizes the resulting infinities in the framework of holographic renormalization. Nevertheless, there is an overlap in the corresponding symmetries.

As described in [49], the algebra of the asymptotic symmetries is the semi-direct sum of the Killing vectors of the unit hyperboloid with a set of super-translations. If we use the following metric on the hyperboloid

$$
h_{a b}^{0} d x^{a} d x^{b}=-\frac{1}{\left(1-s^{2}\right)^{2}} d s^{2}+\frac{1}{\left(1-s^{2}\right)} \bar{\gamma}_{A B} d x^{A} d x^{B},
$$

then the Lorentz algebra is generated by

$$
\mathcal{Y}^{s}=-\left(1-s^{2}\right) b, \quad \mathcal{Y}^{A}=Y^{A}-s \bar{D}^{A} b,
$$

and the abelian algebra of super-translations is parametrized by functions $\omega$ on the hyperboloid satisfying

$$
\left(\mathcal{D}_{a} \mathcal{D}^{a}+3\right) \omega=-\left(1-s^{2}\right)^{2} \partial_{s}^{2} \omega+\left(1-s^{2}\right) \bar{D}_{A} \bar{D}^{A} \omega+3 \omega=0,
$$


where $\mathcal{D}_{a}$ is the covariant derivative associated to the metric $h_{a b}^{0}$. As shown in [49], in the limit $s \rightarrow \pm 1$, the two branches of solutions to the super-translation equation (C.3) have a different behaviour. The one corresponding to odd functions $\widehat{\omega}=\sqrt{1-s^{2}} \omega$ under the combined action of a time reversal and antipodal map tends to finite functions on the sphere and describes the usual BMS super-translations. The algebra of asymptotic symmetries parametrized by (C.2) and (C.3) with odd $\widehat{\omega}$ 's is then the usual BMS asymptotic symmetry algebra at null infinity.

More explicitly, the BMS supertranslation parameter $\mathcal{T}\left(x^{A}\right)$ can be obtained through the following construction. An odd function $\omega=\left(1-s^{2}\right)^{-\frac{1}{2}} \widehat{\omega}$ solution to equation (C.3) has a spherical harmonics expansion given by

$$
\widehat{\omega}=\sum_{l m} \omega_{l, m} \Psi_{l}(s) Y_{l, m}\left(x^{A}\right), \quad \Psi_{l}=\frac{1}{2}\left(1-s^{2}\right)^{2} \partial_{s}^{2} Q_{l} .
$$

The functions $Q_{l}(s)$ are Legendre functions of the second kind and can be written in terms of Legendre polynomials $P_{l}(s)$ as

$$
Q_{l}(s)=P_{l}(s) \frac{1}{2} \log \left(\frac{1+s}{1-s}\right)+\widetilde{Q}_{l}(s)
$$

where $\widetilde{Q}_{l}(s)$ are polynomials. The action of the Lorentz algebra on $\widehat{\omega}$ is given by

$$
\delta_{Y, b} \widehat{\omega}=Y^{A} \partial_{A} \widehat{\omega}-s b \widehat{\omega}-s \bar{D}^{A} b \partial_{A} \widehat{\omega}-\left(1-s^{2}\right) b \partial_{s} \widehat{\omega} .
$$

Defining the BMS supertranslation parameter $\mathcal{T}\left(x^{A}\right)=\lim _{s \rightarrow 1} \widehat{\omega}$, we can evaluate the above identity at $s=1$ using the asymptotic behaviour $\psi_{l}(s)=1+O(1-s)$ to obtain

$$
\delta_{Y, b} \mathcal{T}=Y^{A} \partial_{A} \mathcal{T}-b \mathcal{T}-\bar{D}^{A} b \partial_{a} \mathcal{T}
$$

This is the usual action of a Lorentz transformation on BMS supertranslations.

The difference between the description of the BMS algebra given in (5.3)-(5.6) and the description given in equation (C.7) is in the choice of representative functions used to parametrize the super-translations $\omega$. In order to recover the ADM description of the supertranslation, we have to define $W$ and $T$ as initial conditions at $s=0$ :

$$
\left.\omega\right|_{s=0}=\left.\widehat{\omega}\right|_{s=0}=W\left(x^{A}\right),\left.\quad \partial_{s} \omega\right|_{s=0}=\left.\partial_{s} \widehat{\omega}\right|_{s=0}=T\left(x^{A}\right) .
$$

We see that the hyperboloid function $\widehat{\omega}$ is odd if and only if $W$ and $T$ are respectively an odd and an even function on the sphere. From the action of Lorentz algebra on $\widehat{\omega}$ given in equation (C.6), we can derive the corresponding action on $W$ and $T$ :

$$
\begin{aligned}
\delta_{Y, b} T & =Y^{A} \partial_{A} T-\bar{D}^{A} b \partial_{A} W-b \bar{D}_{A} \bar{D}^{A} W-3 b W, \\
\delta_{Y, b} W & =Y^{A} \partial_{A} W-b T .
\end{aligned}
$$

The change of basis from the pair of functions $(W, T)$ to the BMS supertranslation parameter $\mathcal{T}$ is obtained by solving equation (C.3) with the initial condition (C.8) and then defining

$$
\mathcal{T}=\lim _{s \rightarrow 1}\left(\sqrt{1-s^{2}} \omega\right)
$$


Expending all quantities in spherical harmonics and using the general solution written in (C.4), we can write this change of basis explicitly

$$
\begin{aligned}
& \mathcal{T}=\sum_{l m} \omega_{l, m} Y_{l, m}\left(x^{A}\right), \\
& W=\sum_{k} \sum_{m=-2 k-1}^{2 k+1} W_{2 k+1, m} Y_{2 k+1, m}, \quad T=\sum_{k} \sum_{m=-2 k}^{2 k} T_{2 k, m} Y_{2 k, m}, \\
& \left.\omega_{2 k+1, m} \psi_{2 k+1}\right|_{s=0}=W_{2 k+1, m},\left.\quad \omega_{2 k, m} \partial_{s} \psi_{2 k}\right|_{s=0}=T_{2 k, m} .
\end{aligned}
$$

The first few $\psi_{l}$ functions can be easily computed

$$
\psi_{0}=s, \quad \psi_{1}=1, \quad \psi_{2}=\frac{3}{4}\left(1-s^{2}\right)^{2} \log \left(\frac{1+s}{1-s}\right)+\frac{5}{2} s\left(1-s^{2}\right)
$$

and we can use them to write the first few component of the change of basis:

$$
\omega_{0,0}=T_{0,0}, \quad \omega_{1, m}=W_{1, m}, \quad \omega_{2, m}=\frac{1}{4} T_{2, m} .
$$

Open Access. This article is distributed under the terms of the Creative Commons Attribution License (CC-BY 4.0), which permits any use, distribution and reproduction in any medium, provided the original author(s) and source are credited.

\section{References}

[1] P.A.M. Dirac, Forms of Relativistic Dynamics, Rev. Mod. Phys. 21 (1949) 392 [InSPIRE].

[2] V.A. Fock, The Theory of Space, Time and Gravitation, 1st edition, GITTL, Moscow (1955); 2nd revised edition, Pergamon Press, Oxford (1964).

[3] P.A.M. Dirac, The theory of gravitation in Hamiltonian form, Proc. Roy. Soc. Lond. A $\mathbf{2 4 6}$ (1958) 333 [INSPIRE].

[4] P.A.M. Dirac, Fixation of coordinates in the Hamiltonian theory of gravitation, Phys. Rev. 114 (1959) 924 [INSPIRE].

[5] R.L. Arnowitt, S. Deser and C.W. Misner, The dynamics of general relativity, in Gravitation: an introduction to current research, L. Witten, ed., Wiley, New York (1962), chapter 7, pp. 227-264 [Gen. Rel. Grav. 40 (2008) 1997] [gr-qc/0405109] [INSPIRE].

[6] T. Regge and C. Teitelboim, Role of Surface Integrals in the Hamiltonian Formulation of General Relativity, Annals Phys. 88 (1974) 286 [InSPIRE].

[7] H. Bondi, M.G.J. van der Burg and A.W.K. Metzner, Gravitational waves in general relativity. 7. Waves from axisymmetric isolated systems, Proc. Roy. Soc. Lond. A 269 (1962) 21 [INSPIRE].

[8] R.K. Sachs, Gravitational waves in general relativity. 8. Waves in asymptotically flat space-times, Proc. Roy. Soc. Lond. A 270 (1962) 103 [INSPIRE].

[9] R. Sachs, Asymptotic symmetries in gravitational theory, Phys. Rev. 128 (1962) 2851 [INSPIRE]. 
[10] E.T. Newman and T.W.J. Unti, Behavior of Asymptotically Flat Empty Spaces, J. Math. Phys. 3 (1962) 891 [INSPIRE].

[11] R. Penrose, Asymptotic properties of fields and space-times, Phys. Rev. Lett. 10 (1963) 66 [INSPIRE].

[12] R. Penrose, Zero rest mass fields including gravitation: Asymptotic behavior, Proc. Roy. Soc. Lond. A 284 (1965) 159 [INSPIRE].

[13] T. Mädler and J. Winicour, Bondi-Sachs Formalism, Scholarpedia 11 (2016) 33528 [arXiv: 1609.01731] [INSPIRE].

[14] F. Alessio and G. Esposito, On the structure and applications of the Bondi-Metzner-Sachs group, Int. J. Geom. Meth. Mod. Phys. 15 (2017) 1830002 [arXiv:1709.05134] [INSPIRE].

[15] T. Banks, A critique of pure string theory: Heterodox opinions of diverse dimensions, hep-th/0306074 [INSPIRE].

[16] G. Barnich and C. Troessaert, Symmetries of asymptotically flat 4 dimensional spacetimes at null infinity revisited, Phys. Rev. Lett. 105 (2010) 111103 [arXiv:0909.2617] [INSPIRE].

[17] G. Barnich and C. Troessaert, Aspects of the BMS/CFT correspondence, JHEP 05 (2010) 062 [arXiv: 1001.1541] [INSPIRE].

[18] G. Barnich and C. Troessaert, Supertranslations call for superrotations, PoS (CNCFG2010) 010 [arXiv: 1102 .4632] [INSPIRE].

[19] G. Barnich and C. Troessaert, BMS charge algebra, JHEP 12 (2011) 105 [arXiv:1106.0213] [INSPIRE].

[20] G. Barnich and C. Troessaert, Comments on holographic current algebras and asymptotically flat four dimensional spacetimes at null infinity, JHEP 11 (2013) 003 [arXiv:1309.0794] [INSPIRE].

[21] É.É. Flanagan and D.A. Nichols, Conserved charges of the extended Bondi-Metzner-Sachs algebra, Phys. Rev. D 95 (2017) 044002 [arXiv:1510.03386] [INSPIRE].

[22] G. Barnich and C. Troessaert, Finite BMS transformations, JHEP 03 (2016) 167 [arXiv: 1601.04090] [INSPIRE].

[23] A. Strominger and A. Zhiboedov, Superrotations and Black Hole Pair Creation, Class. Quant. Grav. 34 (2017) 064002 [arXiv: 1610.00639] [INSPIRE].

[24] G. Barnich, Centrally extended BMS4 Lie algebroid, JHEP 06 (2017) 007 [arXiv: 1703.08704] [INSPIRE].

[25] M. Campiglia and A. Laddha, Asymptotic symmetries and subleading soft graviton theorem, Phys. Rev. D 90 (2014) 124028 [arXiv:1408.2228] [InSPIRE].

[26] M. Campiglia and A. Laddha, New symmetries for the Gravitational S-matrix, JHEP 04 (2015) 076 [arXiv : 1502.02318] [INSPIRE].

[27] M. Campiglia and A. Laddha, Sub-subleading soft gravitons: New symmetries of quantum gravity?, Phys. Lett. B 764 (2017) 218 [arXiv:1605.09094] [INSPIRE].

[28] M. Campiglia and A. Laddha, Sub-subleading soft gravitons and large diffeomorphisms, JHEP 01 (2017) 036 [arXiv: 1608. 00685] [INSPIRE].

[29] A. Ashtekar, Asymptotic Quantization of the Gravitational Field, Phys. Rev. Lett. 46 (1981) 573 [INSPIRE]. 
[30] A. Ashtekar, Asymptotic Quantization: Based On 1984 Naples Lectures, Naples, Italy, Bibliopolis (1987), Monographs and Textbooks in Physical Science 2 [INSPIRE].

[31] A. Strominger, On BMS Invariance of Gravitational Scattering, JHEP 07 (2014) 152 [arXiv:1312.2229] [INSPIRE].

[32] T. He, V. Lysov, P. Mitra and A. Strominger, BMS supertranslations and Weinberg's soft graviton theorem, JHEP 05 (2015) 151 [arXiv:1401.7026] [INSPIRE].

[33] F. Cachazo and A. Strominger, Evidence for a New Soft Graviton Theorem, arXiv: 1404.4091 [INSPIRE].

[34] A. Strominger and A. Zhiboedov, Gravitational Memory, BMS Supertranslations and Soft Theorems, JHEP 01 (2016) 086 [arXiv:1411.5745] [INSPIRE].

[35] S. Pasterski, A. Strominger and A. Zhiboedov, New Gravitational Memories, JHEP 12 (2016) 053 [arXiv : 1502.06120] [INSPIRE].

[36] M. Campiglia and A. Laddha, Asymptotic symmetries of gravity and soft theorems for massive particles, JHEP 12 (2015) 094 [arXiv: 1509.01406] [INSPIRE].

[37] G. Compère and J. Long, Vacua of the gravitational field, JHEP 07 (2016) 137 [arXiv: 1601.04958] [INSPIRE].

[38] E. Conde and P. Mao, BMS Supertranslations and Not So Soft Gravitons, JHEP 05 (2017) 060 [arXiv: 1612.08294] [INSPIRE].

[39] S.W. Hawking, M.J. Perry and A. Strominger, Soft Hair on Black Holes, Phys. Rev. Lett. 116 (2016) 231301 [arXiv:1601.00921] [INSPIRE].

[40] G. Compère and J. Long, Classical static final state of collapse with supertranslation memory, Class. Quant. Grav. 33 (2016) 195001 [arXiv:1602.05197] [InSPIRE].

[41] G. Compère, Bulk supertranslation memories: a concept reshaping the vacua and black holes of general relativity, Int. J. Mod. Phys. D 25 (2016) 1644006 [arXiv:1606.00377] [InSPIRE].

[42] S.W. Hawking, M.J. Perry and A. Strominger, Superrotation Charge and Supertranslation Hair on Black Holes, JHEP 05 (2017) 161 [arXiv: 1611.09175] [INSPIRE].

[43] R. Bousso and M. Porrati, Soft Hair as a Soft Wig, Class. Quant. Grav. 34 (2017) 204001 [arXiv: 1706.00436] [INSPIRE].

[44] A. Strominger, Black Hole Information Revisited, arXiv: 1706.07143 [INSPIRE].

[45] R. Bousso and M. Porrati, Observable Supertranslations, Phys. Rev. D 96 (2017) 086016 [arXiv: 1706. 09280] [INSPIRE].

[46] A. Strominger, Lectures on the Infrared Structure of Gravity and Gauge Theory, arXiv: 1703.05448 [INSPIRE].

[47] R. Beig and N. oMurchadha, The Poincaré group as the symmetry group of canonical general relativity, Annals Phys. 174 (1987) 463 [INSPIRE].

[48] G. Compère and F. Dehouck, Relaxing the Parity Conditions of Asymptotically Flat Gravity, Class. Quant. Grav. 28 (2011) 245016 [Erratum ibid. 30 (2013) 039501] [arXiv:1106. 4045] [INSPIRE].

[49] C. Troessaert, The BMS4 algebra at spatial infinity, Class. Quant. Grav. 35 (2018) 074003 [arXiv: 1704.06223] [INSPIRE]. 
[50] J. Corvino and R.M. Schoen, On the asymptotics for the vacuum Einstein constraint equations, J. Diff. Geom. 73 (2006) 185 [gr-qc/0301071] [INSPIRE].

[51] L.-H. Huang, Solutions of special asymptotics to the Einstein constraint equations, Class. Quant. Grav. 27 (2010) 245002 [arXiv: 1002.1472] [INSPIRE].

[52] J. Corvino and D. Pollack, Scalar Curvature and the Einstein Constraint Equations, arXiv: 1102.5050 [INSPIRE].

[53] R. Benguria, P. Cordero and C. Teitelboim, Aspects of the Hamiltonian Dynamics of Interacting Gravitational Gauge and Higgs Fields with Applications to Spherical Symmetry, Nucl. Phys. B 122 (1977) 61 [inSPIRE].

[54] M. Henneaux and C. Teitelboim, Asymptotically anti-de Sitter Spaces, Commun. Math. Phys. 98 (1985) 391 [INSPIRE].

[55] C.W. Misner, The Flatter regions of Newman, Unti and Tamburino's generalized Schwarzschild space, J. Math. Phys. 4 (1963) 924 [inSPIRE].

[56] C.W. Bunster, S. Cnockaert, M. Henneaux and R. Portugues, Monopoles for gravitation and for higher spin fields, Phys. Rev. D 73 (2006) 105014 [hep-th/0601222] [INSPIRE].

[57] C. Troessaert, Hamiltonian surface charges using external sources, J. Math. Phys. 57 (2016) 053507 [arXiv: 1509.09094] [INSPIRE].

[58] M. Bianchi, D.Z. Freedman and K. Skenderis, How to go with an RG flow, JHEP 08 (2001) 041 [hep-th/0105276] [INSPIRE].

[59] M. Bianchi, D.Z. Freedman and K. Skenderis, Holographic renormalization, Nucl. Phys. B 631 (2002) 159 [hep-th/0112119] [INSPIRE].

[60] M. Henneaux, C. Martinez, R. Troncoso and J. Zanelli, Black holes and asymptotics of 2+1 gravity coupled to a scalar field, Phys. Rev. D 65 (2002) 104007 [hep-th/0201170] [INSPIRE].

[61] M. Henneaux, C. Martinez, R. Troncoso and J. Zanelli, Asymptotically anti-de Sitter spacetimes and scalar fields with a logarithmic branch, Phys. Rev. D 70 (2004) 044034 [hep-th/0404236] [INSPIRE].

[62] M. Henneaux, C. Martinez, R. Troncoso and J. Zanelli, Asymptotic behavior and Hamiltonian analysis of anti-de Sitter gravity coupled to scalar fields, Annals Phys. $\mathbf{3 2 2}$ (2007) 824 [hep-th/0603185] [INSPIRE].

[63] J.D. Brown and M. Henneaux, On the Poisson Brackets of Differentiable Generators in Classical Field Theory, J. Math. Phys. 27 (1986) 489 [inSPIRE].

[64] H. Friedrich, Gravitational fields near space-like and null infinity, J. Geom. Phys. 24 (1998) 83.

[65] H. Friedrich and J. Kannar, Bondi type systems near space-like infinity and the calculation of the NP constants, J. Math. Phys. 41 (2000) 2195 [gr-qc/9910077] [INSPIRE].

[66] H. Friedrich and J. Kannar, Calculating asymptotic quantities near space-like and null infinity from Cauchy data, Annalen Phys. 9 (2000) 321 [gr-qc/9911103] [INSPIRE].

[67] M. Campiglia and R. Eyheralde, Asymptotic U(1) charges at spatial infinity, JHEP 11 (2017) 168 [arXiv: 1703.07884] [INSPIRE].

[68] A. Strominger, Asymptotic Symmetries of Yang-Mills Theory, JHEP 07 (2014) 151 [arXiv: 1308.0589] [INSPIRE]. 
[69] G. Barnich and P.-H. Lambert, Einstein-Yang-Mills theory: Asymptotic symmetries, Phys. Rev. D 88 (2013) 103006 [arXiv:1310.2698] [INSPIRE].

[70] A. Ashtekar and R.O. Hansen, A unified treatment of null and spatial infinity in general relativity. I - Universal structure, asymptotic symmetries and conserved quantities at spatial infinity, J. Math. Phys. 19 (1978) 1542 [InSPIRE].

[71] R. Beig and B. Schmidt, Einstein's equations near spatial infinity, Commun. Math. Phys. 87 (1982) 65.

[72] R. Beig, Integration Of Einstein's Equations Near Spatial Infinity, Proc. Roy. Soc. Lond. A 391 (1984) 295.

[73] O. Baghchesaraei, R. Fareghbal and Y. Izadi, Flat-Space Holography and Stress Tensor of Kerr Black Hole, Phys. Lett. B 760 (2016) 713 [arXiv: 1603.04137] [INSPIRE]. 